\title{
Prevalence of Pulmonary Complications in Patients with Rheumatoid Arthritis
}

\author{
Lamyaa Al Sa'idi ${ }^{1}$, Nasser Al Busaidii ${ }^{2}$, Humaid Al Wahshi ${ }^{3}$, Farida Al Balushi ${ }^{4}$ \\ ${ }^{1}$ Sohar Hospital, Sultanate of Oman; Internal medicine department \\ ${ }^{2}$ Royal Hospital, Muscat, Sultanate of Oman; Chest department \\ ${ }^{3}$ Royal Hospital, Muscat, Sultanate of Oman; Rheumatology department \\ ${ }^{4}$ Royal Hospital, Muscat, Sultanate of Oman; Rheumatology department \\ *Corresponding Author: Nasser Al Busaidi, Royal Hospital, Muscat, Sultanate of Oman; Chest \\ department, Oman.
}

\begin{abstract}
Objectives: to investigate the prevalence of pulmonary complications including ILD, bronchiectasis, pleural effusion, rheumatoid nodule and pulmonary hypertension in patients with RA. In addition to look for variables that might be associated with the development of pulmonary complications.

Method: This is a retrospective study that included all Omani patients who were diagnosed with RA based on 2010 ACR/EULAR criteria. All patients were selected from Royal hospital (a tertiary care center, Muscat, Oman) from January 2006 to October 2015. The complications were assessed based on radiological imaging and echocardiography; HRCT for ILD, bronchiectasis and rheumatoid nodule, CXR for pleural effusion and Echocardiography for Pulmonary hypertension. Prevalence of pulmonary complications was recorded and association with different variables was assessed.

Results: A total of 662 patients had fulfilled 2010 ACR/EULAR criteria for RA. 129 (19.5\%) patients had pulmonary complications whereas 533 (88.5\%) did not. Some patients were found to have single pulmonary complication whereas others had multiple complications. The most prevalent complication in Omani patients was bronchiectasis which accounted for $11.2 \%$ followed by ILD $10.9 \%$ with some variables being statistically significant based on $P$ value such as the age. The rest of pulmonary complications were pleural effusion $1.8 \%$, rheumatoid nodule $1.4 \%$ and pulmonary hypertension account for $3.6 \%$.
\end{abstract}

Conclusion: Bronchiectasis and ILD were found to be the most prevalent pulmonary manifestations in Omani patients with $R A$.

Keywords: Rheumatoid arthritis, interstitial Lung disease, Pulmonary Hypertension, Disease Modifying Anti Rheumatic Drugs, pulmonary complications.

\section{Study Limitations}

This study is good as it is the first study to be conducted in Oman which has investigating the prevalence of pulmonary complications in patients with RA. It has large sample size. However, since it is a retrospective study it has some limitations including missing data in terms of history (such as symptoms duration, smoking history, family history, date of the diagnosis and previous visits in other hospital). Also there is missing investigations in the initial visits such as RF and anti CCP. Furthermore, this study was done in one center only which is Royal hospital.

Another important limitation to consider is that we did not exclude patients with preexisting pulmonary diseases like asthma and COPD and we did not assess the severity index for RA using CRP 28 score and other scores. This could be partly due to missing data as well.

Abbreviations

- - RA : Rheumatoid Arthritis

- - ILD : Interstitial Lung Disease

- - PH: Pulmonary Hypertension

- -HRCT: High Resolution Computed Tomography

- -ACR/EULAR: American college of Rheumatology/ European League Against Rheumatism

- - CRP : C reactive Protein

- - ESR : Erythrocyte Sedimentation Rate

- - RF : Rheumatoid Factor 
- -Anti CCP : Anti Cyclic Citrullinated Peptide

- -DMARD : Disease Modifying Anti Rheumatic Drugs

- - US : United State

\section{INTRODUCTION}

Rheumatoid Arthritis is a chronic systemic autoimmune inflammatory disorder that affects mainly the joints resulting in erosive destruction. It is associated with autoantibodies production mainly Rheumatoid Factor and Anti Cyclic citrullinated peptide antibody. A study was conducted in US showed that RA affects nearly $12-70$ per 100,000 patients a year in men and $25-130$ per 100,000 in women ${ }^{(1-3)}$.

There is a variety of extra articular manifestations that occur in $\mathrm{RA}^{(4-5)}$. Pulmonary manifestations are considered a major cause leading of morbidity and mortality in patients with RA ${ }^{(6)}$. The lung can be directly affected by the course of the disease or secondary as a result of DMARD or biologic agents which are used for treatment of RA ${ }^{(7-9)}$.

According to M. Shaw et al, Wang J.-X. et al studies, interstitial lung disease is the most common pulmonary complication in patients with RA which usually predicts poor prognosis $(6-7,17)$. It's prevalence usually ranges from 7 $10 \% .^{(10-11)}$ Some studies have investigated the association between autoantibodies such as anti CCP and ILD. Inui et al. study did not find an association between anti-CCP and ILD whereas Nikiphorou et al demonstrated that anti-CCP antibodies were strongly associated with ILD in RA ${ }^{(12-15)}$. Another multicentre study which was conducted in UK found that Anti CCP is strongly associated with RA- ILD and smoking was strongly associated with ILD in men. ${ }^{(16)}$

In a study conducted by Attar et al in Saudi Arabia, 35 out of 100 patients with RA developed bronchiectasis with multiple variables being major predictors for development of bronchiectasis such as age, male gender, disease activity and anti CCP. ${ }^{(18-20)}$.

Other pulmonary manifestations that have been studied previously include pleural effusion, rheumatoid nodule, and pulmonary Hypertension ${ }^{(21-22)}$. Antin Ozerki et al has found that pleural effusion is more common in men than women and it's incidence ranged from 3\% - $22 \%^{(21-23)}$. Same study has found that some factors were associated with increase the risk of pleural effusion including being a male gender, smoking, high RF titre, HLA/Dw3 HLA antigen, low serum complement and late onset disease ${ }^{(24)}$. The above quoted study also showed that the incidence of pulmonary nodule incidence can be as low as $2 \%$ with CXR and can be as high as $22 \%$ with using highly sensitive modality like HRCT ${ }^{(23-24)}$. In a metaanalysis looking at the prevalence of pulmonary hypertension in connective tissue diseases, two studies by Dowson et al and Keser et al reported prevalence of Pulmonary hypertension in RA patients ranging from $21-27.5 \%{ }^{(25-27)}$.

Unfortunately, there is no single previous study done to look for prevalence of pulmonary complications of RA among Omani patients. The objective of this study is to carry out a descriptive retrospective study to investigate for the prevalence of pulmonary complications and to look for variables which may predict the development of these complications in all patients who were diagnosed to have RA based on ACR/EULAR 2010 criteria and were on regular follow up in rheumatology and chest clinic in Royal Hospital from January 2006 to October 2015.

\section{METHOD}

A retrospective study was conducted at tertiary care centre Royal Hospital (Muscat Region, Sultanate of Oman). The study was approved by research and Ethics Committee in Royal Hospital. A total of 1153 patients' records were screened. We included all Omani patients 12 years or above who were diagnosed to have RA based on 2010 ACR/EULAR classification system with pulmonary involvement during period from January 2006 to October 2015. All patients had to be on regular follow up in chest and rheumatology clinics in RH. Non-Omani patients and those who were not on regular follow up (has 1 or 2 visit to the hospital) were excluded from the study. A total of 662 patients have fulfilled the above criteria.

In this study we focused on 5 main pulmonary complications which were ILD, bronchiectasis, pleural effusion, rheumatoid nodule and pulmonary hypertension.

Data were collected from patient's clinical records including demographic features (e.g age, gender), clinical findings (e.g age at diagnosis, disease duration, smoking history) and treatment history ( non-biologic DMARDs, biologic agents) ( Table 1). The laboratory parameters were considered as well including $\mathrm{C}$ reactive protein (normal level 0-5), erythrocyte sedimentation rate, Rheumatoid factor (normal level 0-28), and Anti CCP (normal level 0-5). (Table 1) 
Table1. Demographic characteristics of the patients

\begin{tabular}{|c|c|c|}
\hline Characteristic & Frequency (\%) & Mean (SD) \\
\hline Age & & $48.9(13.883)$ \\
\hline Male & $81(12.2 \%)$ & \\
\hline Female & $581(87.8 \%)$ & \\
\hline Age at Diagnosis & & $42.4(13.86)$ \\
\hline Disease Duration & & $6.49(4.29)$ \\
\hline Smoker & $35(5.3 \%)$ & \\
\hline Non Smoker & $620(93.7 \%)$ & \\
\hline Previous Smoker & $7(1.1 \%)$ & \\
\hline CRP at Diagnosis & & $22.2(39.00)$ \\
\hline ESR at Diagnosis & & $39.12(26.41)$ \\
\hline RF titer & & $201(138.18)$ \\
\hline Anti CCP titer & & $126.2(77.7)$ \\
\hline Biologic treatment & $237(35.8 \%)$ & \\
\hline No biologic treatment & $425(64.2 \%)$ & \\
\hline
\end{tabular}

Presence of pulmonary complications was assessed based on imaging studies and echocardiography. HRCT was used to assess presence of ILD, bronchiectasis and rheumatoid nodule. CXR was used to assess presence of pleural effusion whereas Echocardiography was used to assess for pulmonary hypertension (defined as pulmonary artery systolic pressure of > $35 \mathrm{mmhg}$ based on echocardiography).

Data were collected from patient's records using Al Shifa system based on ICD 10 disease classification. Data was collected initially in data collection sheet then were transferred to Epi data software. Data were analysed using
Statistical package for social sciences SPSS 20 software. Descriptive statistics were used for mean and standard deviations and frequency distribution for count and percentages. Results were considered significant when $\mathrm{P}$ value was less than 0.05 .

\section{RESULTS}

A total of 662 patients fulfilled the 2010 ACR/EULAR criteria for diagnosis of RA. A total of $81(12.2 \%)$ were males whereas 581 $(87.8 \%)$ were females. Out of 662 patients, a total of $129(19.5 \%)$ patients had either one or more pulmonary complications. (Figure1).

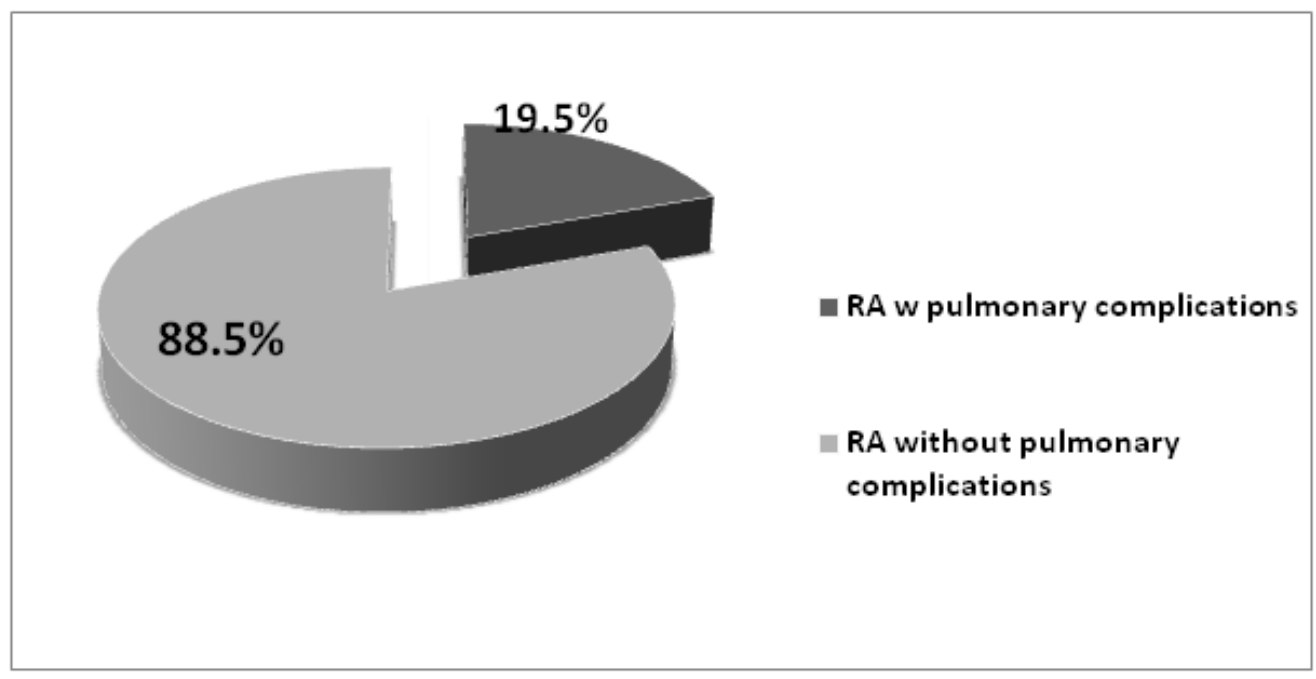

Figure1. Percentage of patients with RA with/without pulmonary complications

The mean age for patients was 48.9 years with mean disease duration of 6.5 years. 72 patients $(10.9 \%)$ were found to have Interstitial lung disease based on HRCT and 74 patients (11.2\%) who were found to have bronchiectasis based on same diagnostic modality. These two complications were found to be the most prevalent pulmonary complications among
Omani patients with RA. There were 12 patients (1.8\%) with pleural effusion based on CXR, 9 patients $(1.4 \%)$ with pulmonary nodules based on HRCT and 24 patients (3.6\%) with pulmonary hypertension based on echocardiography (Figure 2). Around 80 (12\%) patients had a single complication and 49 (7.4\%) patients had more than one. Out of those 
$30(4.5 \%)$ patients were found to have ILD with bronchiectasis. 4 patients had bronchiectasis and pulmonary hypertension, 3 patients had ILD and
Pulmonary hypertension and 2 patients had combined ILD, bronchiectasis and Pleural effusion.

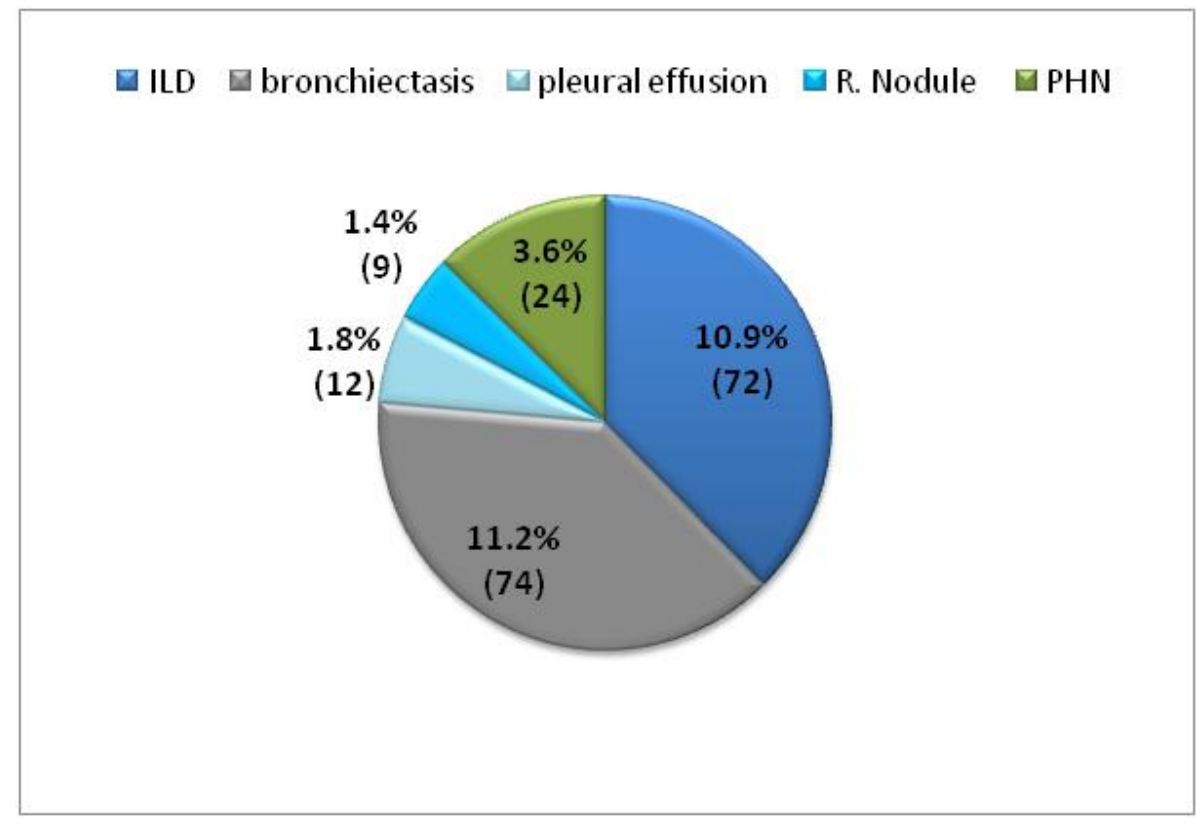

Figure2. Prevalence of pulmonary complications among RA patients

On further analysis, the following variables were studied in relation to development of pulmonary complications: age, gender, disease duration, smoking history, CRP, ESR, positive Rheumatoid factor, Anti CCP and use of biologic and non-biologic DMARDs.

Overall, there was a statistically significant difference $(\mathrm{P}=0.0001)$ in the mean age between those who had pulmonary complications (58.86 +/- 11.86) and those who did not $(46.49+$ +/13.26). The older age group has a higher risk of having pulmonary complications. Furthermore, pulmonary complications were found to be more common among patients who are on biological agents " $\mathrm{P}=0.0001$.

There was a statistically significant association between development of ILD and the following variables: age $(\mathrm{P}=0.0001), \mathrm{CRP}(\mathrm{P}=0.029)$, $\mathrm{ESR}$ $(\mathrm{P}=0.002)$, smoking history $(\mathrm{P}=0.036)$ and biological treatment $(\mathrm{P}=0.001)$. In addition, following variables were found associated with developing bronchiectasis: age $(\mathrm{P}=0.0001), \mathrm{CRP}$ $(\mathrm{P}=0.029), \quad \mathrm{ESR} \quad(\mathrm{P}=0.047)$ and biologic treatment $(\mathrm{P}=0.001)$. Age was the only variable with statistical significant association for the development of both pleural effusion and Pulmonary Hypertension ( $\mathrm{P}=0.0001)$ whereas age $(\mathrm{P}=0.002)$ and biological treatment $(\mathrm{P}=0.012)$ were found to be associated with development of rheumatoid nodules. The study did not prove any significant association between gender, positive RF, Anti CCP and development of pulmonary complications (Table 2).

Table2. Statistically significant variables for predicting pulmonary Complications

\begin{tabular}{|c|c|c|}
\hline Pulmonary complications & Variables & P value \\
\hline \multirow{4}{*}{ ILD } & Age & 0.0001 \\
\cline { 2 - 3 } & CRP & 0.029 \\
\cline { 2 - 3 } & ESR & 0.002 \\
\cline { 2 - 3 } & Smoking & 0.036 \\
\cline { 2 - 3 } & DMARDs & 0.001 \\
\hline \multirow{2}{*}{ Bronchiectasis } & Age & 0.0001 \\
\cline { 2 - 3 } & CRP & 0.029 \\
\cline { 2 - 3 } & ESR & 0.047 \\
\cline { 2 - 3 } & DMARDs & 0.001 \\
\hline Pleural effusion & Age & 0.0001 \\
\hline Rheumatoid nodules & Age & 0.002 \\
\cline { 2 - 3 } & DMARDs & 0.012 \\
\hline Pulmonary Hypertension & Age & 0.0001 \\
\hline
\end{tabular}




\section{DISCUSSION}

This study demonstrates that ILD and bronchiectasis are the most prevalent pulmonary complications in patients with RA. According to study conducted in Japan, the prevalence of ILD was $7.7-12 \%$ which is almost similar to our results ${ }^{(7)}$.

Additionally, we found that several factors including age, CRP, ESR, smoking history, and biological treatment correlated significantly with development of ILD in RA patients. The significance of biologic DMARDs could be explained because of disease severity as these agents are usually started in severe RA or it could be related to the drugs themselves. There were some cases reported in which using biologic agents has caused rapid progression of ILD ${ }^{(7,29-30)}$. In our cohortthere are only 3 patients $(0.45 \%)$ were found to have ILD likely associated with non-biological DMARDs (such as Methotrexate) as documented in their files however these were not confirmed by biopsy. There was no correlation between serology (anti CCP, positive RF) and ILD found in our study cohort and these findings are similar to study by Inui et al ${ }^{(12,14)}$. On the other hand, other studies including Nikiphorou et al and another study conducted in Mexico by Rocha-Muñoz had found that anti CCP was strongly associated with presence and extent of ILD in patients with $\mathrm{RA}^{(12,15)}$. The differences between studies might be contributed to the differences in patient populations, disease definition and methods were used for evaluating and detecting the clinical parameters ${ }^{(13)}$. The differences in results between studies can be explained, that studies have used different parameters to assess severity of ILD such as 6MWT, Borg scales, decrease in FVC\% and severity of ILD in HRCT ${ }^{(12)}$.

In our study, the prevalence of bronchiectasis among Omani patients with RA was $11.2 \%$, age, CRP, ESR, and biological treatment found to be the major parameters associated with development of bronchiectasis in patients with RA. Around 30 patients $(4.5 \%)$ were found to have coexistence of both ILD and bronchiectasis.

A study conducted in Saudi Arabia, found that $35 \%$ of patients with moderately active RA had developed bronchiectasis with some variables such as age, male gender, disease duration and anti CCP being major association for its development ${ }^{(18)}$. However, population was only 100 patients, all these patients with moderate active RA, whereas our cohort has large number of patients with mixture of disease severity ranging from mild to severe disease, and this could explain the differences in the prevalence of bronchiectasis among the two populations.

In a study conducted by Antin-Ozerkis showed that pleural manifestations of RA are more common in older patients and male gender as well ${ }^{(24)}$. It stated that the incidence of pleural effusion in most studies ranged between 3-5\% and could be as high as $22 \%$ with males being affected predominantly ${ }^{(24)}$. In comparison to other studies that used both CXR and HRCT for detecting small pleural effusion, we used CXR alone. This can contribute to the difference in the prevalence of pleural effusion between the two studies, Another possible reason for the low prevalence of pleural effusion in this study is that the vast majority of patients in this study were females "females to males ratio was 7:1" Since male gender was previously shown to be associated with the development of pleural effusion, this may explain the low prevalence of pleural effusions in our study.

In one study by Antin Ozerkis, it was found that rheumatoid nodules prevalence was $2 \%$ based on CXR and can be up to $22 \%$ with using high sensitive imaging modality such HRCT ${ }^{(24)}$. In our cohort, the prevalence of the above complication is slightly lower.

Moreover, Udayakumar et al study had compared 45 patients with RA with 45 patients who were healthy and found that there is higher risk of developing asymptomatic pulmonary hypertension in patients with RA $26.7 \%$ compared with the matched controls $(4.5 \%)^{(4,28)}$. Furthermore, the same study has found that older patients and those with longer disease duration are more likely to develop the above complication ${ }^{(4,28)}$. This can support our finding that older patients are likely to develop pulmonary hypertension. The same study found that 3 patients $(6.7 \%)$ had coexistence of pulmonary hypertension with ILD ${ }^{(28)}$. In our study we found only 3 patients $(0.45 \%)$ with both ILD and pulmonary hypertension.

The strength of our study lies in its being the first study to be conducted in Oman which has investigating the prevalence of pulmonary complications in patients with RA. It has large sample size. However, it has some limitations including missing data in terms of history (such as symptoms duration, smoking history, family history, date of the diagnosis and previous visits in other hospital).Also there is missing investigations in the initial visits such as RF and 
anti CCP. Furthermore, this study was done in one center only which is Royal hospital.

Another important limitation to consider is that we did not exclude patients with preexisting pulmonary diseases like asthma and COPD and we did not assess the severity index for RA using CRP 28 score and other scores. This could be partly due to missing data as well. This study was conducted to get a generalized idea about the prevalence of pulmonary complications in Omani patients with RA. Further studies are needed to study the following: clinical manifestations of each pulmonary complication, respiratory symptoms that can occur in relation with the disease severity, changes in the pulmonary function test in patients with ILD and most common changes in radiological imaging in some complications like ILD and bronchiectasis. Another point that needs to be studied in detail is drug induced pulmonary diseases.

\section{CONCLUSION}

Pulmonary diseases are one of the extra articular complications that are considered to be a major cause of morbidity and mortality in patients with Rheumatoid arthritis. In Omani population, we have found that Bronchiectasis and ILD are the most prevalent complications and this can be related to disease process or the use of biologic medications. These complications are more common in elderly patients with longer disease duration. Different variables have been found to correlate with these complications and can be used as predictors for early detection and management.

\section{REFERENCES}

[1] Solomon JJ, Ryu JH, Tazelaar HD, Myers JL, Tuder R, Cool CD, et al. Fibrosing interstitial pneumonia predicts survival in patients with rheumatoid arthritis-associated interstitial lung disease (RA-ILD). Respir Med. 2013 Aug;107(8):1247-52.

[2] Turesson C, Matteson EL. Management of extra-articular disease manifestations in rheumatoid arthritis. Curr Opin Rheumatol May 2004;16(3):206e11.

[3] Gabriel SE, Crowson CS, Kremers HM, et al. Survival in rheumatoid arthritis: a populationbased analysis of trends over 40years. Arthritis Rheum Jan 2003;48(1):54e8.

[4] Shaw M, Collins BF, Ho LA, Raghu G. Rheumatoid arthritis-associated lung disease. Eur Respir Rev. 2015 Mar;24(135):1-16.

[5] Marigliano B, Soriano A, Margiotta D, et al. Lung involvement in connective tissue diseases: a comprehensive review and a focus on rheumatoid arthritis. Autoimmun Rev 2013; 12: $1076-1084$.

[6] O’Dwyer DN, Armstrong ME, Cooke G, et al. Rheumatoid arthritis (RA) associated interstitial lung disease (ILD). Eur J Intern Med 2013; 24: 597-603.

[7] Mori S. Management of Rheumatoid Arthritis Patients with Interstitial Lung Disease: Safety of Biological Antirheumatic Drugs and Assessment of Pulmonary Fibrosis. Clin Med Insights Circ Respir Pulm Med. 2015 Sep;41.

[8] 8.Roubille C1, Haraoui B2. Interstitial lung diseases induced or exacerbated by DMARDS and biologic agents in rheumatoid arthritis: a systematic literature review.Semin Arthritis Rheum. 2014 Apr;43(5):613-26. doi: 10.1016/ j.semarthrit.2013.09.005. Epub 2013 Oct 5.

[9] Gauhar UA, Gaffo AL, Alarcon GS. Pulmonary manifestations of rheumatoid arthritis. Semin Respir Crit Care Med 2007;28(4):430-40.

[10] Y. Yin, D. Liang, L. Zhao et al., "Anti-cyclic citrullinated peptide antibody is associated with interstitial lung disease in patients with rheumatoid arthritis," PLoS ONE, vol. 9, no. 4, Article ID e92449, 2014.

[11] Bongartz, T. et al. Incidence and mortality of interstitial lung disease in rheumatoid arthritis: a population-based study. Arthritis Rheum. 62, 1583-1591 (2010).

[12] Rocha-Muñoz AD, Ponce-Guarneros M, Gamez-Nava JI, Olivas-Flores EM, Mejía M, Juárez-Contreras P, et al. Anti-Cyclic Citrullinated Peptide Antibodies and Severity of Interstitial Lung Disease in Women with Rheumatoid Arthritis. J Immunol Res. 2015; 2015:1-10.

[13] Yin Y, Liang D, Zhao L, Li Y, Liu W, Ren Y, et al. Anti-Cyclic Citrullinated Peptide Antibody Is Associated with Interstitial Lung Disease in Patients with Rheumatoid Arthritis. Zheng SG, editor. PLoS ONE. 2014 Apr 17;9(4):e92449.

[14] N. Inui, N. Enomoto, T. Suda, Y. Kageyama, H. Watanabe, and K. Chida, "Anti-cyclic citrullinated peptide antibodies in lung diseases associated with rheumatoid arthritis," Clinical Biochemistry, vol. 41, no. 13, pp. 1074-1077, 2008.

[15] E. Nikiphorou, E. Chan, V. Saravanan et al., "Serological biomarkers for the development of rheumatoid arthritis related interstitial lung disease," Arthritis \& Rheumatology, vol. 65, supplement 10, p. 423, 2013.

[16] C. A. Kelly, V. Saravanan, M. Nisar et al., "Rheumatoid arthritis-related interstitial lung disease: associations, prognostic factors and physiological and radiological characteristicsa large multicentre UK study," Rheumatology (Oxford), vol. 53, no. 9, pp. 1676-1682, 2014. 
[17] Wang T, Zheng X-J, Liang B-M, Liang Z-A. Clinical features of rheumatoid arthritisassociated interstitial lung disease. Sci Rep. 2015 Oct 7;5:14897.

[18] Attar SM, Alamoudi OS, Aldabbag AA. Prevalence and risk factors of asymptomatic bronchiectasis in patients with rheumatoid arthritis at a tertiary care center in Saudi Arabia. Ann Thorac Med. 2015;10(3):176.

[19] Kaushik VV, Hutchinson D, Desmond J, Lynch MP, Dawson JK. Association between bronchiectasis and smoking in patients with rheumatoid arthritis. Ann Rheum Dis 2004; 63:1001-2.

[20] Despaux J, Manzoni P, Toussirot E, Auge B, Cedoz JP,Wendling D. Prospective study of the prevalence of bronchiectasis in rheumatoid arthritis using high-resolution computed tomography. Rev Rhum Engl Ed 1998;65:453-61.

[21] Balbir-Gurman A, Yigla M, Nahir AM, et al. Rheumatoid pleural effusion. Semin Arthritis Rheum 2006;35(6):368-78.

[22] Jurik AG, Davidsen D, Graudal H. Prevalence of pulmonary involvement in rheumatoid arthritis and its relationship to some characteristics of the patients. A radiological and clinical study. Scand J Rheumatol 1982; 11(4):217-24.

[23] Remy-Jardin M, Remy J, Cortet B, et al. Lung changes in rheumatoid arthritis: CT findings. Radiology 1994;193(2):375
[24] Antin-Ozerkis D, Evans J, Rubinowitz A, Homer RJ, Matthay RA. Pulmonary Manifestations of Rheumatoid Arthritis. Clin Chest Med. 2010 Sep; 31(3):451-78.

[25] Yang X, Mardekian J, Sanders KN, Mychaskiw MA, Thomas J. Prevalence of pulmonary arterial hypertension in patients with connective tissue diseases: a systematic review of the literature. Clin Rheumatol. 2013 Oct;32 (10): 1519-31.

[26] Dawson JK et al (2000) Raised pulmonary artery pressures measured with Doppler echocardiography in rheumatoid arthritis patients. Rheumatology (Oxford) 39(12):13201325

[27] Keser G et al (2004) Pulmonary hypertension in rheumatoid arthritis.Scand J Rheumatol 33(4):244-245

[28] Udayakumar N, Venkatesan S, Rajendiran C. Pulmonary hypertension in rheumatoid arthritis - relation with the duration of the disease. Int $\mathbf{J}$ Cardiol 2008; 127: 410-412.

[29] Cannon GW. Methotrexate pulmonary toxicity. Rheum Dis Clin North Am 1997;23(4):917-37.

[30] Schnabel A, Dalhoff K, Bauerfeind S, et al. Sustained cough in methotrexate therapy for rheumatoid arthritis. ClinRheumatol 1996; 15(3):277-82.

Citation: Lamyaa Al Sa'idi et.al, "Prevalence of Pulmonary Complications in Patients with Rheumatoid Arthritis”, International Journal of Research Studies in Medical and Health Sciences. 2020; 5(6): 54-60.

Copyright: (C) 2020 Lamyaa Al Sa'idi et.al, This is an open-access article distributed under the terms of the Creative Commons Attribution License, which permits unrestricted use, distribution, and reproduction in any medium, provided the original author and source are credited. 